\title{
Cholesterol synthesis inhibitor RO 48-8071 suppresses transcriptional activity of human estrogen and androgen receptor
}

\author{
BENFORD MAFUVADZE, YAYUN LIANG and SALMAN M. HYDER \\ Department of Biomedical Sciences and Dalton Cardiovascular Research Center, \\ University of Missouri, Columbia, MO 65211, USA
}

Received May 6, 2014; Accepted June 18, 2014

DOI: $10.3892 /$ or.2014.3332

\begin{abstract}
Breast cancer cells express enzymes that convert cholesterol, the synthetic precursor of steroid hormones, into estrogens and androgens, which then drive breast cancer cell proliferation. In the present study, we sought to determine whether oxidosqualene cyclase (OSC), an enzyme in the cholesterol biosynthetic pathway, may be targeted to suppress progression of breast cancer cells. In previous studies, we showed that the OSC inhibitor RO 48-8071 (RO) may be a ligand which could potentially be used to control the progression of estrogen receptor- $\alpha(\mathrm{ER} \alpha)$-positive breast cancer cells. Herein, we showed, by real-time PCR analysis of mRNA from human breast cancer biopsies, no significant differences in OSC expression at various stages of disease, or between tumor and normal mammary cells. Since the growth of hormone-responsive tumors is ER $\alpha$-dependent, we conducted experiments to determine whether RO affects ER $\alpha$. Using mammalian cells engineered to express human ER $\alpha$ or ER $\beta$ protein, together with an ER-responsive luciferase promoter, we found that RO dose-dependently inhibited $17 \beta$-estradiol (E2)-induced $\mathrm{ER} \alpha$ responsive luciferase activity ( $\mathrm{IC}_{50}$ value, $\sim 10 \mu \mathrm{M}$ ), under conditions that were non-toxic to the cells. RO was less effective against ER $\beta$-induced luciferase activity. Androgen receptor (AR) mediated transcriptional activity was also reduced by RO. Notably, while ER $\alpha$ activity was reduced by atorvastatin, the HMG-CoA reductase inhibitor did not influence AR activity, showing that RO possesses broader antitumor properties. Treatment of human BT-474 breast cancer cells with RO reduced levels of estrogen-induced PR protein, confirming that RO blocks ER $\alpha$ activity in tumor cells. Our
\end{abstract}

Correspondence to: Dr Salman M. Hyder, Department of Biomedical Sciences and Dalton Cardiovascular Research Center, University of Missouri, 134 Research Park Drive, Columbia, MO 65211, USA E-mail: hyders@missouri.edu

Key words: cholesterol inhibitor, oxidosqualene cyclase inhibitor, RO 48-8071, estrogen receptor- $\alpha$, estrogen receptor- $\beta$, androgen receptor findings demonstrate that an important means by which RO suppresses hormone-dependent growth of breast cancer cells is through its ability to arrest the biological activity of ER $\alpha$. This warrants further investigation of RO as a potential therapeutic agent for use against hormone-dependent breast cancers.

\section{Introduction}

It is becoming increasingly apparent that cholesterol and its metabolites contribute to the development of breast cancer (1-3). Various mechanisms by which cholesterol promotes the growth of breast tumors have been identified; cholesterol is also the precursor of steroid hormones such as estrogen and testosterone, both of which have well-recognized tumor promoting effects (4). The cholesterol metabolite 27-hydroxycholesterol has been shown to exert selective estrogen receptor modulation (SERM) effects $(5,6)$ and also to promote the growth of estrogen receptor (ER)-positive breast tumors (3). Current evidence, therefore, suggests that by disrupting cholesterol biosynthesis, we may inhibit cell cycle progression and induce cell death (7). Most previous studies have targeted HMG-CoA reductase, a rate limiting enzyme in the cholesterol biosynthetic pathway. However, using in silico analysis, we recently found that oxidosqualene cyclase (OSC), which is downstream of HMG-CoA reductase and is a critical enzyme that catalyzes the cyclization of 2,3-oxidosqualene to lanosterol, may also be a potential target by which to control the proliferation of ER+ve tumors (8). On the basis of these initial findings, and after examining the effects of different OSC inhibitors on human breast cancer cells, we selected RO 48-8071 (RO) as a prototype inhibitor. Our decision to use RO was also based on the aforementioned study where we showed that the compound is a suitable candidate for suppressing human breast cancer cells in vitro (8).

Since there are differences in intra-tumor estrogen levels between ER+ve and triple negative breast cancer tissue (9), we hypothesized that the levels of enzymes involved in the cholesterol biosynthetic pathway may be altered in breast cancer cells. This could include differential expression or activity of OSC. Taking this into consideration, we measured levels of OSC in a panel of human breast cancers and normal tissues and found no significant difference in levels of OSC 
mRNA between steroid responsive and hormone-independent tumors. Furthermore, OSC expression in tumor tissue was not significantly different than in normal mammary tissue, suggesting that RO must suppress breast cancer cell growth via alternative, off-target effects. In this respect, we discovered that RO suppresses the transcriptional activity of $\mathrm{ER} \alpha$ and to some extent that of ER $\beta$, under conditions that preserve cell viability. Moreover, RO also suppresses androgen receptor (AR) transcriptional activity, another major determinant of breast cancer progression (10). Using western blot analysis, we verified that RO suppresses levels of progesterone receptor (PR), the expression of which is directly controlled by ER $\alpha$ in human breast cancer cells (11). This confirms that the ER $\alpha$-mediated signal transduction pathway is inhibited when cells are exposed to RO.

\section{Materials and methods}

RO 48-8071 was purchased from Sigma, dissolved in DMSO and stored in aliquots at $-20^{\circ} \mathrm{C}$ prior to use.

Human tissue qPCR. qPCR-ready TissueScan ${ }^{\mathrm{TM}}$ cDNA Array (OriGene starter kit, cat. no. TSRT101) was initially used to determine the expression of OSC in human breast cancer tissues following guidelines recommended by the manufacturer. Human breast cancer tissue array (cat. no. BCRT101) was then used to measure OSC expression in tissues that either expressed ER, PR and HER2 (hormone-dependent) or tissues lacking these receptors (triple negative) at different stages of development (stage I-III). Human OSC (Hs00158906_ml LSS) TaqMan Fam probes were obtained from Applied Biosystems and normalized with human GAPDH (Hs03929097_g1 FG). Relative gene expression was determined using the following formula: Fold-change in gene expression, $2-\Delta \Delta \mathrm{Ct}=2-\{\Delta \mathrm{Ct}$ (cancer tissue samples) - $\Delta \mathrm{Ct}$ (normal tissue) $\}$, where $\Delta \mathrm{Ct}=\mathrm{Ct}$ (OSC) - Ct (GAPDH), where Ct represents threshold cycle number.

Luciferase activity. Receptor assay systems were obtained from Indigo Biosciences (State College, PA, USA) and luciferase activity was determined following the manufacturer's recommended protocol. The assays comprised non-human mammalian cells engineered to express human $\operatorname{ER} \alpha, \operatorname{ER} \beta$, androgen receptor (AR) protein and luciferase reporter gene functionally linked to the corresponding nuclear receptor -responsive promoter. We initially used Indigo Biosciences Receptor Assay (cat. no. IB00421-48P) to determine the effects of RO on estradiol-induced $\mathrm{ER} \alpha$ and $\mathrm{ER} \beta$ activity. ICI 182,780 was used as a reference antagonist to $17 \beta$-estradiol. Since our initial results showed more pronounced effects of RO on ER $\alpha$ activity compared with $\mathrm{ER} \beta$, we focused on ER $\alpha$-promoter linked activity using Indigo Biosciences Receptor Assay (cat. no. IB00401). We also determined the effects of a commonly used cholesterol inhibitor, atorvastatin (Ator) on ER $\alpha$ promoter-linked activity. Since there is increasing evidence to support the role of AR in the development of breast cancer (10), we conducted studies to assess the effects of RO on AR-dependent luciferase activity. A human AR reporter assay system (cat. no. IB03001; Indigo Biosciences) was used with $6 \alpha-F 1$ testosterone as the reference agonist for AR. For all reporter studies, we sequentially used fluorescence-based LCM assays (cat. no. LCM-01; Indigo Biosciences), following the manufacturer's guidelines, to determine the relative number of live cells at the assay endpoint. All luciferase assays were read with GloMax ${ }^{\circledR}$-Multi+ Microplate Multimode Reader system (Promega).

Western blotting. BT-474 breast cancer cells were grown in a humidified atmosphere of $5 \% \mathrm{CO}_{2}$ at $37^{\circ} \mathrm{C}$ in $100-\mathrm{mm}$ cell culture plates using phenol red-free Dulbecco's modified Eagle's medium (DMEM/F12) supplemented with $10 \%$ fetal bovine serum. When cells reached 50-60\% confluence, they were washed with PBS and switched into DMEM/F12 supplemented with 5\% dextran-coated charcoal (DCC) for $24 \mathrm{~h}$. Cells were then washed, transferred into fresh DMEM/F12 and treated with E2 $(10 \mathrm{nM})$ in the absence and presence of RO $(5 \mu \mathrm{M})$ or with RO alone. Cells were treated with $\mathrm{RO}$ for $3 \mathrm{~h}$ prior to treatment with E2 for $16 \mathrm{~h}$, after which cells were harvested. Nuclear protein was extracted following the manufacturer's guidelines (cat. no. 40010; Active Motif, USA). Protein aliquots $(20 \mu \mathrm{g})$ were separated by SDS-PAGE, transferred to polyvinylidene difluoride membrane and blotted with the following human specific antibodies: ER $\alpha$ (SC-8005, 1:200 dilution), ER $\beta$ (SC-8974, 1:200 dilution), PR (SC-810, 1:200 dilution) (all from Santa Cruz Biotechnology), $\beta$-actin (Sigma-Aldrich, St. Louis, MO, USA). Protein bands were detected and quantified by blotting with anti-mouse secondary antibody (SC-2005, 1:10,000 dilution), or anti-rabbit secondary antibody (SC-2004, 1:10,000 dilution) (both from Santa Cruz Biotechnology), and a chemiluminescent detection system according to the manufacturer's instructions (Amersham Pharmacia Biotech).

Real-time PCR. Total RNA was extracted from two distinct breast cancer cell lines, BT-474 and T47D cells, which express both $\mathrm{ER} \alpha$ and $\mathrm{ER} \beta$. Cultured cells were treated with $20 \mu \mathrm{M}$ RO for 1, 3, 6 and $24 \mathrm{~h}$, respectively. Control samples were treated with ethanol, the vehicle medium in which RO was dissolved. RNA was extracted using an EZ-Bioresearch RNA Isolation kit (cat. no. R1002-50) according to the manufacturer's instructions. Two micrograms of RNA were reverse transcribed into cDNA using a high capacity DNA synthesis kit (cat. no. 4368814; Applied Biosystems). cDNA was then amplified using an ABI 7300 Real-Time PCR Instrument (Applied Biosystems), specific TaqMan primers and TaqMan ${ }^{\circledR}$ Universal PCR Master Mix. Human ER $\alpha$ (Hs00174860_m1), ER- $\beta$ (Hs00230957_m1) and GAPDH (Hs03929097_g1 FG) TaqMan Fam probes were used (Applied Biosystems). Relative gene expression was determined using the following formula: Fold-change in gene expression, $2-\Delta \Delta \mathrm{Ct}=2-\{\Delta \mathrm{Ct}$ (treated samples) - $\Delta \mathrm{Ct}$ (untreated control samples) $\}$, where $\Delta \mathrm{Ct}=\mathrm{Ct}$ $(\mathrm{ER} \alpha$ or $\mathrm{ER} \beta)-\mathrm{Ct}(\mathrm{GAPDH})$, where $\mathrm{Ct}$ represents threshold cycle number. All reactions were carried out in duplicate for at least three independent experiments.

Statistical analysis. Comparisons were made between multiple groups by analysis of variance (ANOVA) with Neuman-Keuls post-hoc testing. Where normality was not achieved, non-parametric ANOVA (Kruskal-Wallis) and post-hoc Dunn's test was performed. Significance was defined as $p<0.05$. Unless indicated, values are shown as mean \pm SEM. 


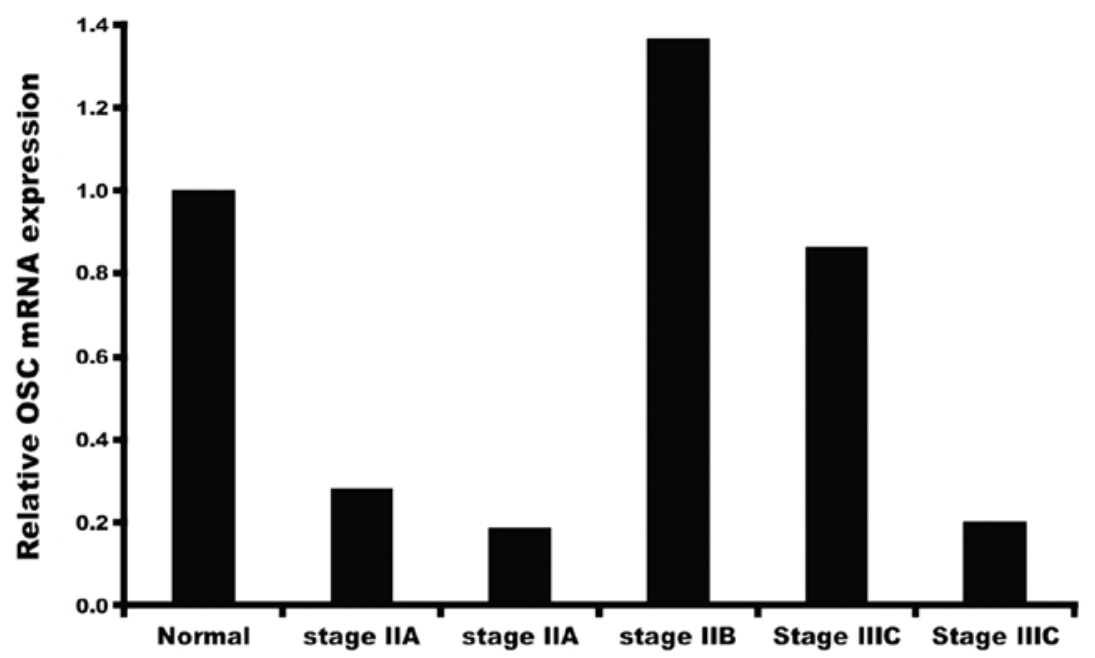

Figure 1. Relative OSC mRNA expression in breast cancer at different stages of growth. Human qPCR-ready TissueScan ${ }^{\mathrm{TM}}$ cDNA Arrays (cat. no. TSRT101) were obtained from OriGene and real-time PCR was conducted as described in Materials and methods. Results showed variable levels of expression of OSC mRNA within different tumors compared with corresponding normal tissue. OSC, oxidosqualene cyclase.

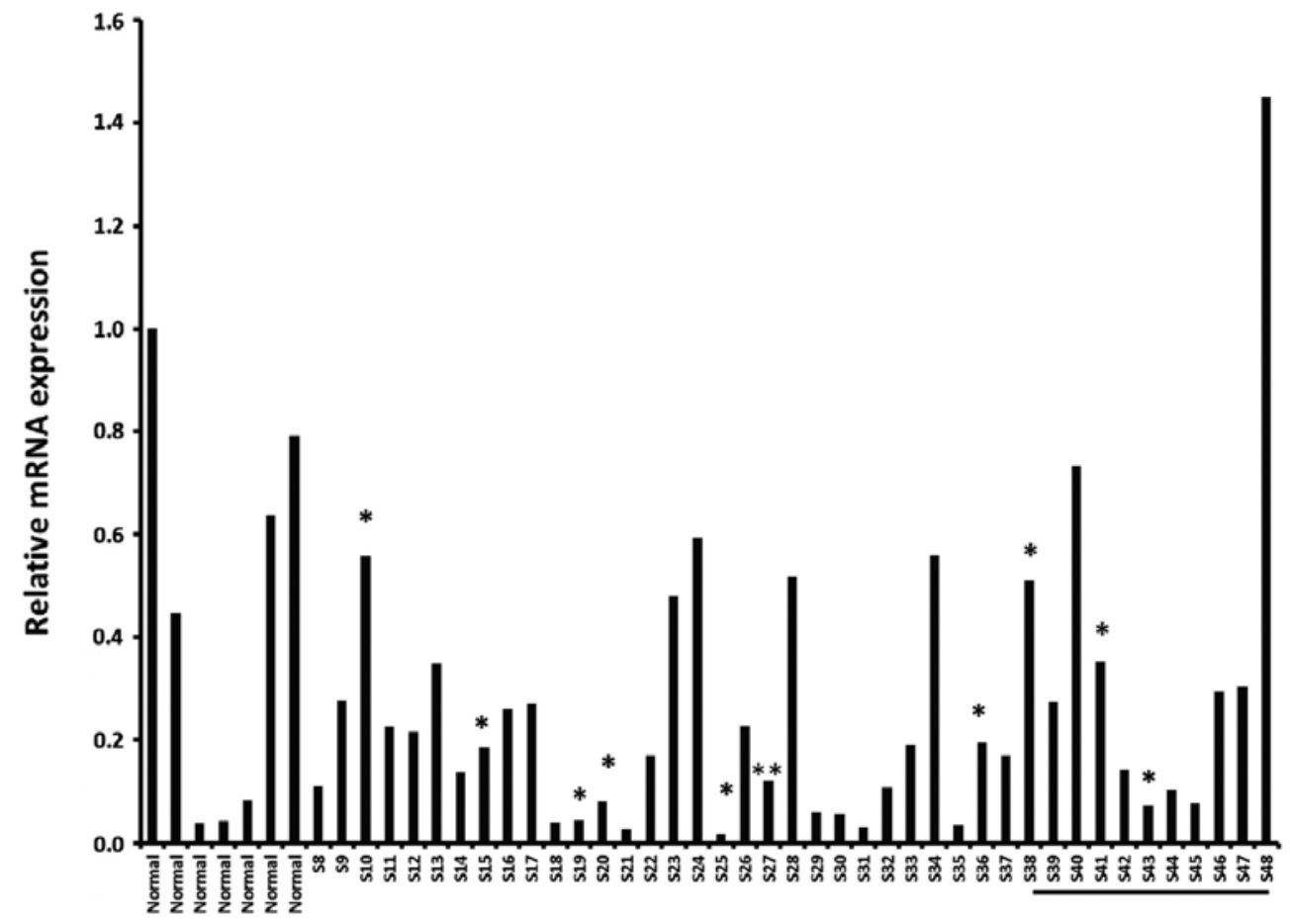

Figure 2. Relative OSC mRNA expression in distinct breast cancer tissues at different stages of growth (stage I-III). Human qPCR-ready TissueScan ${ }^{\mathrm{TM}}$ cDNA Arrays (cat. no. BCRT101) were obtained from OriGene and real-time PCR was conducted as described in Materials and methods. Results showed no significant difference in OSC mRNA expression between hormone-dependent $\left(\mathrm{ER}^{+}, \mathrm{PR}^{+}, \mathrm{HER} 2^{+}\right)$and triple negative breast cancer at various stages of growth "Triple negative breast tumor, ${ }^{* *}$ ER/PR-negative/Her-2neu high tumors; remaining bars represent ER/PR positive tumors. OSC, oxidosqualene cyclase.

\section{Results}

OSC mRNA expression in human cancer tissues. In order to measure OSC expression we employed ready to use qRT-PCR human tissue cDNA arrays to obtain preliminary data on levels of OSC mRNA in a limited number of tumors collected at various stages of development (Fig. 1). Our initial study showed that varying levels of OSC message are present in samples of tumor obtained at different stages, and that, depending on the sample, mRNA levels may be higher or lower than levels present in normal breast tissue. This prompted us to analyze a larger cohort of samples and to focus on different breast cancer tissues at different stages (stage I-III) available from OriGene as described in Materials and methods. As shown in Fig. 2, there was no consistency in levels of OSC expression, either within tissue from normal breast, or within breast tumor samples collected at various stages, including triple negative cancers. 
A

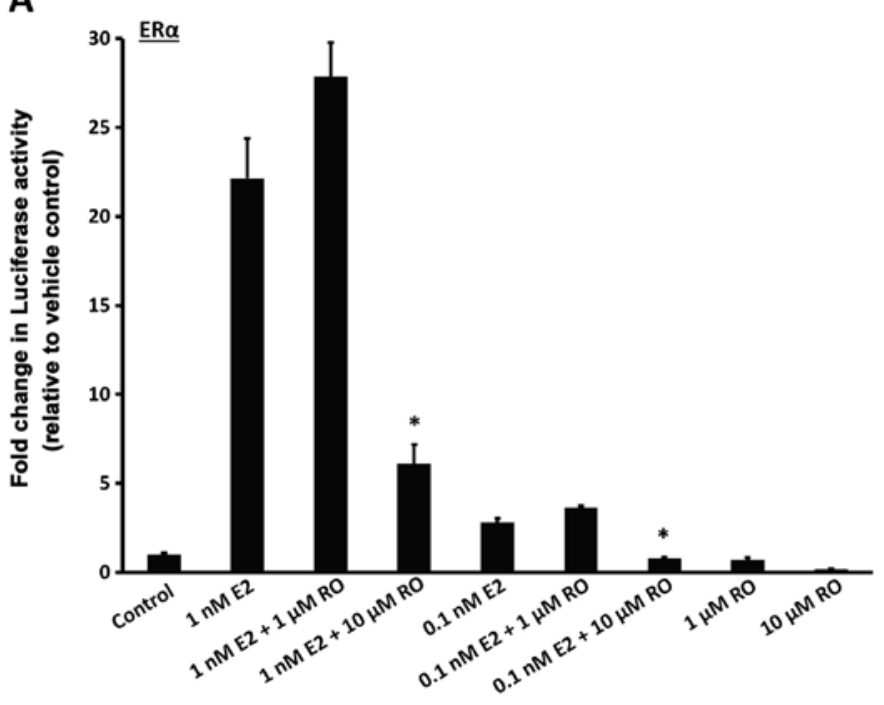

B

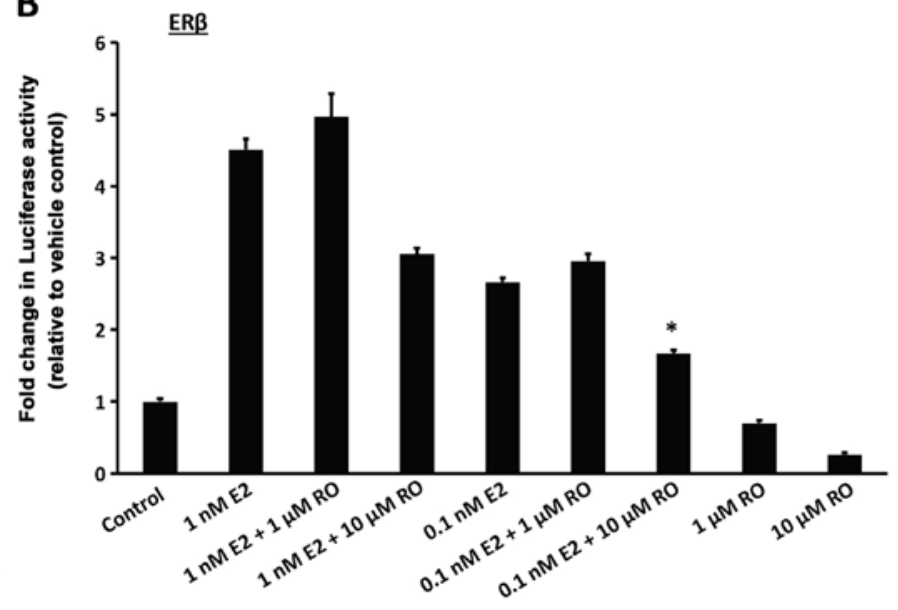

c

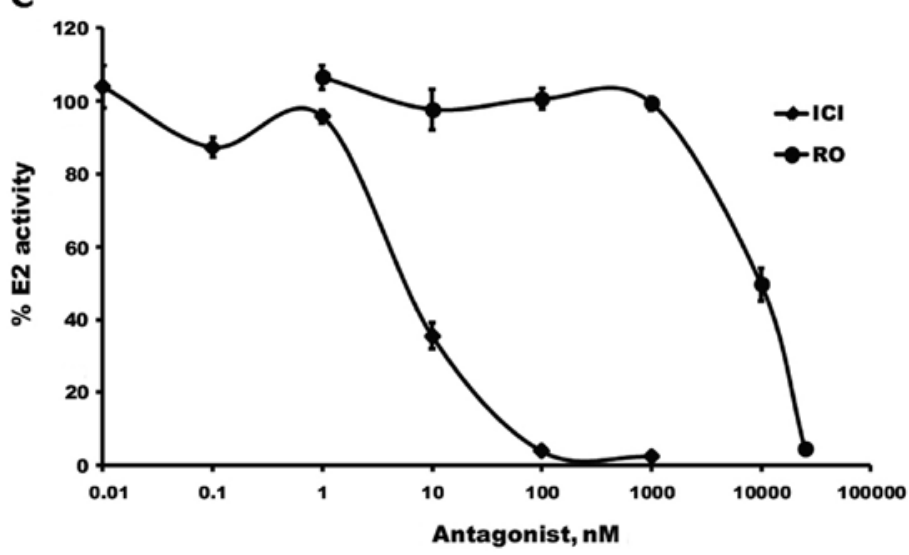

D

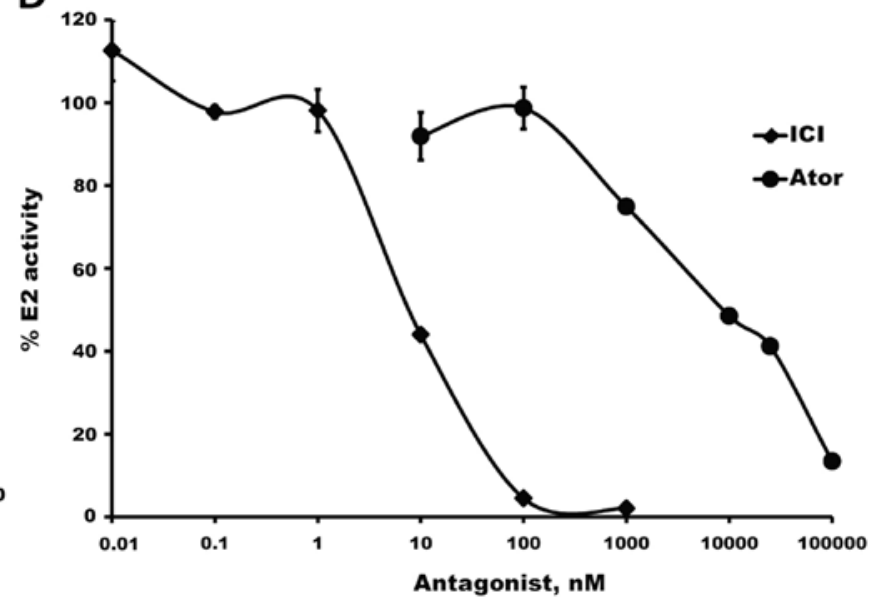

Figure 3. RO significantly inhibits 17ß-estradiol induced ER-mediated luciferase activity. Non-human mammalian cells were engineered with human (A) ERo and (B) ER $\beta$ expression plasmid and luciferase reporter gene functionally linked to estrogen response element. Cells were seeded in 96-well plates according to the manufacturer's protocol. Cells were treated with 0.1 or $1 \mathrm{nM} 17 \beta$-estradiol (E2) \pm 1 or $10 \mu \mathrm{M}$ RO for $18 \mathrm{~h}$, after which luciferase activity was determined Results showed that $10 \mu \mathrm{M}$ RO significantly inhibited E2-induced luciferase activity with both ER $\alpha$ and ER $\beta .{ }^{*} \mathrm{p}<0.05$, significantly different from group treated with similar concentration of E2. (C) RO dose-dependent inhibition of ER $\alpha$-mediated luciferase activity. Cells were seeded in 96-well plates as described above and treated with $1 \mathrm{nM}$ E2 alone or in combination with either ICI 182,780 (ICI) at concentrations varying from 0.01-1,000 $\mathrm{nM}$ or RO at concentrations between 1.0 and 50,000 $\mathrm{nM}$. Results are expressed as percentage of maximum luciferase activity obtained with $1 \mathrm{nM}$ E2. (D) Atorvastatin (Ator) dose-dependently inhibited ER $\alpha$-linked luciferase activity. Cells were seeded in 96-well plates as described above and treated with $1 \mathrm{nM}$ E2 alone or in combination with either ICI at concentrations varying from 0.01-1,000 nM or Ator at concentrations between 1.0-100,000 nM. Results are expressed as percentage of $1 \mathrm{nM}$ E2 activity. RO, RO 48-8071.

Effects of $R O$ on $E R \alpha, E R \beta$ and AR-promoter linked hormone responsive luciferase activity. In a previous study, it was indicated that RO reduces breast cancer cell viability in a dose-responsive manner (8). However, since we found levels of OSC mRNA in breast tumors to be inconsistent (Figs. 1 and 2), we concluded that it was unlikely that RO exerts its effects on breast cancer cells primarily by targeting OSC. Previously, we showed that pharmacological levels of RO degrade ER $\alpha$ in breast cancer cells (Liang et al unpublished data). In the present study, we examined whether lower levels of the drug also influenced the transcriptional activity of ER, without degrading the receptor. For these studies, we used steroid receptor-driven luciferase reporter assays (Indigo Biosciences) and concentrations of RO that did not affect cell viability, as determined from a previous study (8), or ER $\alpha$ protein levels. We found that treatment with $1 \mathrm{nM} 17 \beta$-estradiol for $22 \mathrm{~h}$ increased luciferase activity in both ER $\alpha$ and ER $\beta$-linked reporter cells (Fig. 3A and B; bars on left). Induction was much stronger with $\mathrm{ER} \alpha$ than ER $\beta$, an observation in accordance with a previous study (12). RO caused a decrease in E2-mediated transcriptional response in a manner that was dose-dependent. Both $\mathrm{ER} \alpha$ and $\mathrm{ER} \beta$ luciferase activities were reduced in response to RO (Fig. 3A and B), although RO-mediated transcriptional suppression was much greater with $10 \mu \mathrm{M}$ RO when ER $\alpha$ activity was assessed $(>50 \%)$ than when ER $\beta$ induced transcription was measured $(<50 \%)$.

Having examined the effects of RO on transcriptional activity mediated by both ER $\alpha$ and ER $\beta$, we used ICI 182,780, a well-characterized ER $\alpha$ antagonist, to specifically suppress ER $\alpha$-mediated transcription (Fig. 3C). ICI 182,780 inhibited 


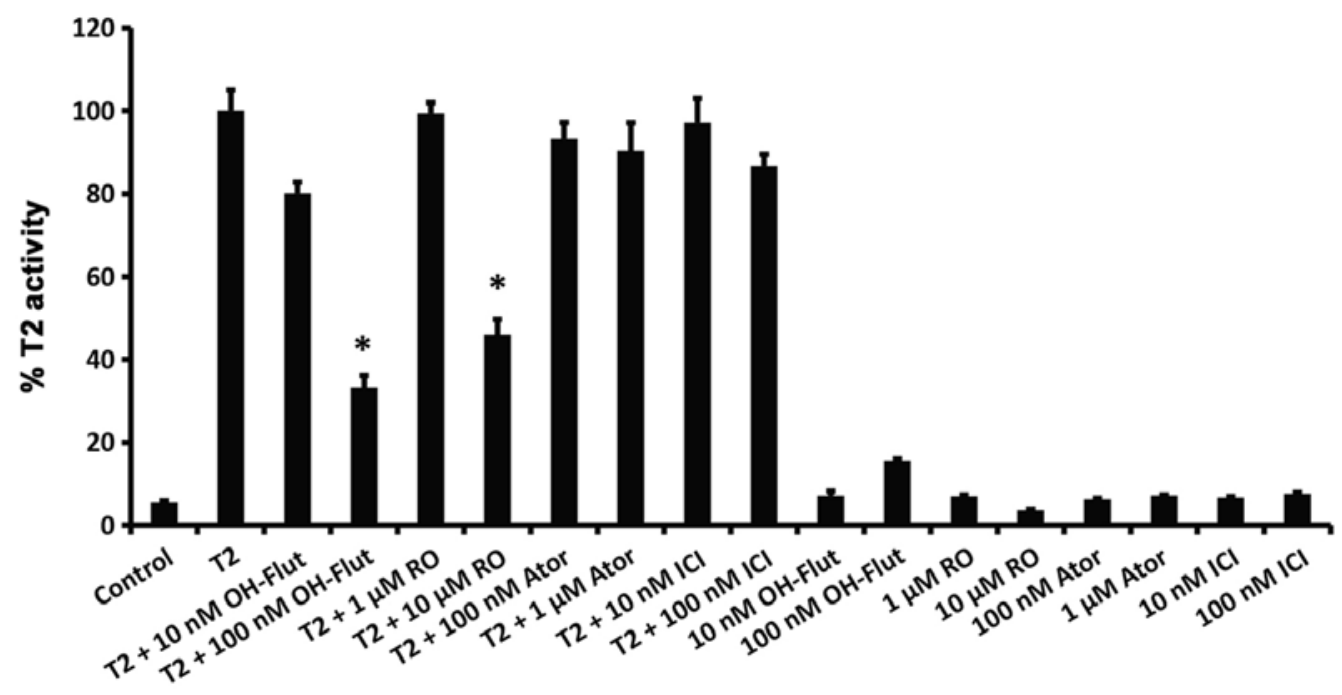

Figure 4. RO significantly inhibits $6 \alpha$-testosterone-induced AR linked luciferase activity. Non-human mammalian cells were engineered to express human AR protein and luciferase reporter gene functionally linked to corresponding AR-responsive luciferase promoter. Cells were seeded in 96 -well plates and treated with $400 \mathrm{pM} 6 \alpha$-testosterone (T2) alone or in combination with one of the following at the concentrations given below; RO (1 or $10 \mu \mathrm{M})$, ICI (10 or $100 \mathrm{nM}$ ), Ator $(100 \mathrm{nM}$ or $1 \mu \mathrm{M})$ or flutamide $(\mathrm{OH}$-flut) $(10$ or $100 \mathrm{nM})$, or the various compounds alone. After $18 \mathrm{~h}$, luciferase activity was determined following the manufacturer's guidelines. Results showed that both RO and $\mathrm{OH}$-flut significantly inhibited T2 induced AR-linked luciferase activity in a dose-dependent manner while ICI and Ator did not affect luciferase activity. * p<0.05, significantly different from T2 group. RO, RO 48-8071; AR, androgen receptor; Ator, atorvastatin.

transcriptional activity considerably more potently than RO $(\sim 1,000$-fold), although, notably, atorvastatin, another cholesterol biosynthesis inhibitor that acts on $\mathrm{HMG}-\mathrm{CoA}$ reductase and is commonly used in humans to lower cholesterol levels, inhibited ER $\alpha$-mediated transcriptional activity at a level comparable to RO (Fig. 3D).

Markedly, RO also inhibited a $6 \alpha$-testosterone-mediated increase in AR transcriptional activity, which was also blocked by the AR antagonist hydroflutamide (OH-flut; Fig. 4). However, in contrast to their suppression of ER $\alpha$-mediated transcriptional activity (Fig. 3), neither atorvastatin nor ICI 182,780 had any inhibitory effect on AR-mediated transcription (Fig. 4).

In order to ensure that the observed reduction in luciferase reporter activity was not due to induced cell apoptosis, a fluorescence-based LCM assay kit (LCM-01; Indigo Biosciences) was used concurrently to measure the proportion of live cells in wells where luciferase activity was measured. As shown in Fig. 5, while the proportion of live cells was significantly reduced by the apoptosis-inducing compound staurosporine, all the other compounds tested in the present study were non-toxic at the concentrations used.

Effect of RO on estradiol-induced PR expression in BT-474 human breast cancer cells. Confirmation that RO was able to modify the biological activity of ER $\alpha$ was obtained in BT-474 cells in which ER $\alpha$ regulates PR levels by increasing PR gene transcription (11). As shown in Fig. 6A, treatment of BT-474 cells with $10 \mathrm{nM}$ E2 for $16 \mathrm{~h}$ resulted in increased levels of both PRA and PRB. E2-mediated increases in PR levels were blocked by RO $(5 \mu \mathrm{M})$, without any significant loss of ER $\alpha$. Real-time PCR confirmed that $5 \mu \mathrm{M}$ RO had no effect on ER $\alpha$ mRNA expression in human BT-474 or T47-D breast cancer cells (Fig. 6B).

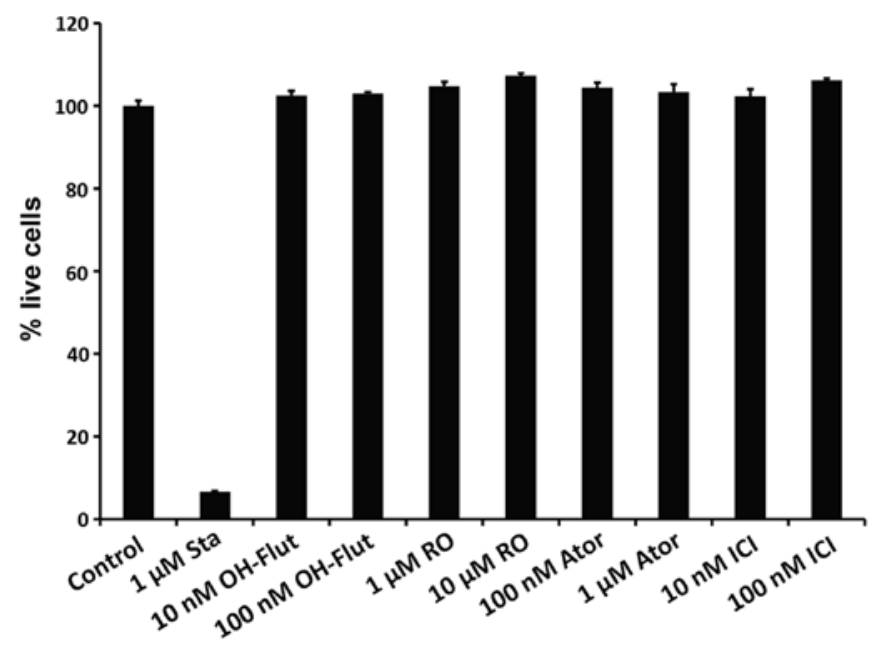

Figure 5. RO and other compounds tested in the present study do not show cytotoxic effects at the concentrations used. A fluorescence-based LCM assay kit (LCM-01; Indigo Biosciences) was used concurrently to assess the proportion of live cells in wells where luciferase activity was measured. Staurosporine $(4 \mu \mathrm{M})$ was used as a positive control for apoptosis and cell loss. RO, RO 48-8071.

\section{Discussion}

There is growing evidence to suggest that cholesterol and its metabolites play an important role in the development of breast cancer (1-3). Previous studies showed that inhibition of cholesterol synthesis at different enzymatic steps of the biosynthetic pathway leads to a suppression of cell growth $(13,14)$, although the significance of OSC in tumor progression remains unclear. OSC is a critical enzyme which is involved in the cyclization of 2,3-oxidosqualene to lanosterol and an increase in its 

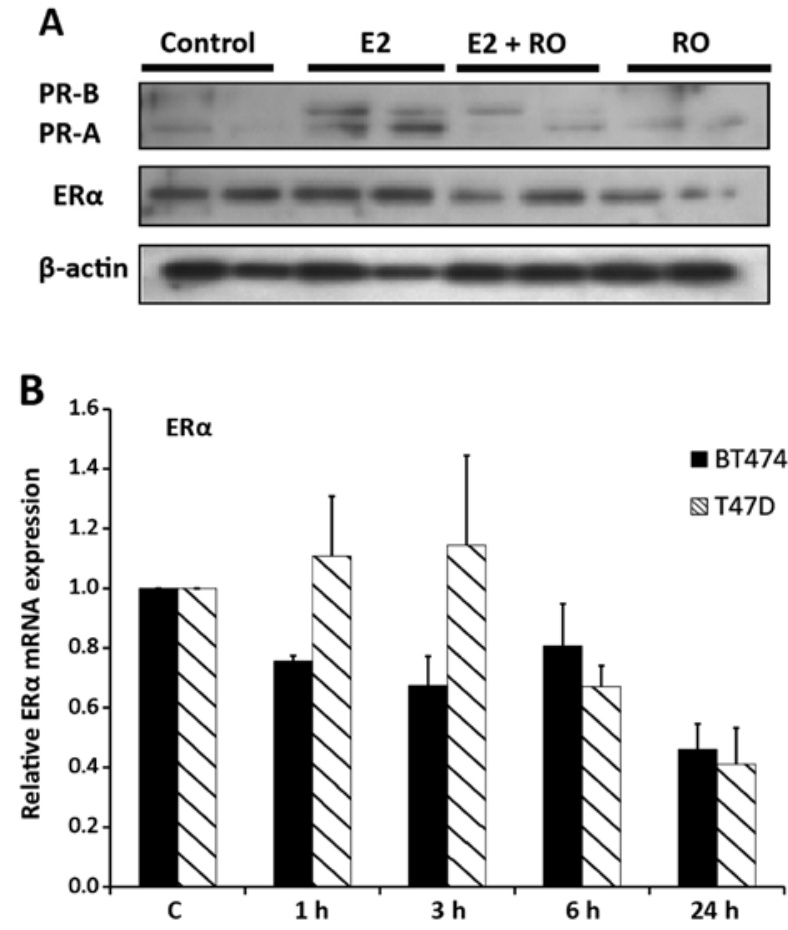

Figure 6. (A) RO inhibits $17 \beta$-estradiol-induced PR protein expression in BT-474 human breast cancer cells. BT-474 cells were treated with either DMSO (vehicle medium) or $5 \mu \mathrm{M}$ RO for $3 \mathrm{~h}$ prior to treatment with $10 \mathrm{nM}$ $17 \beta$-estradiol for $16 \mathrm{~h}$. Protein was then extracted and western blot analysis performed as described in Materials and methods. Results showed that RO reduced E2-induced levels of PR protein. (B) Relative ER $\alpha$ mRNA expression in human BT-474 and T47-D breast cancer cells treated with RO as described in Materials and methods. Results showed no significant changes in ER $\alpha$ mRNA expression. RO, RO 48-8071.

intra-tumor expression or activity has the potential to raise the levels of cholesterol metabolites with estrogenic activities. Such an increase could result in subsequent proliferative effects within breast cancer cells. In earlier studies involving in silico analysis, we showed that RO, an inhibitor of cholesterol synthesis that targets OSC, is a potent ligand with chemotherapeutic properties, which reduces breast cancer cell viability (8). This led us to investigate whether RO may have potential as a chemotherapeutic agent against a broader range of breast cancer cells.

Our initial goal in the present study was to determine whether hormone-responsive and hormone-independent tumors express OSC differently. We hypothesized that differences in OSC expression may partially account for both increased cholesterol biosynthesis and higher levels of intracellular estrogen, which could promote tumor growth. After extensive analysis of a variety of tumor tissues collected at different stages of development and from varying types of tumor, we concluded that there were no significant differences in levels of OSC expression between normal, hormone responsive, hormone-independent and triple-negative breast cancers. It remains to be established, however, whether OSC protein levels are different between the various types of tumor. Based on these observations, we concluded that expression of OSC is unlikely to be a prognostic marker for breast cancer.

In our previous study, we showed that RO significantly reduced the viability of $\mathrm{ER} \alpha$-positive breast cancer cells (8,
Liang et al submitted). However, since levels of OSC expression did not vary between different tumor types, we concluded that it is unlikely that the ability of RO to disrupt tumor cell proliferation is due entirely to its inhibition of OSC. Since $\mathrm{ER} \alpha$ is a major determinant for human breast cancer cell proliferation, we conducted studies to determine whether RO targets ER $\alpha$, initially using non-human mammalian cells engineered to express human $\mathrm{ER} \alpha$ protein, and luciferase reporter gene functionally linked to an ER $\alpha$-responsive promoter to assess $17 \beta$-estradiol induced luciferase activity. Changes in luciferase expression in cells treated with $\mathrm{RO}$ and other test compounds provided a sensitive surrogate measure of changes in $\mathrm{ER} \alpha$ transcriptional activity without cellular toxicity. As expected, ICI 182,780, a well characterized antagonist which acts partially through downregulation of the receptor (15), blocked the effects of $17 \beta$-estradiol on ER $\alpha$. RO also inhibited $17 \beta$-induced ER $\alpha$-linked luciferase expression in a dosedependent manner, although higher doses of $\mathrm{RO}$ were required to achieve levels of inhibition comparable to ICI 182,780 (Figs. 3 and 4). RO also blocked ER $\beta$ activity, although less potently than its inhibition of ER $\alpha$. Notably, atorvastatin, an alternative inhibitor of cholesterol biosynthesis which inhibits HMG-CoA reductase, also blocked ER $\alpha$-mediated transcriptional activity. To determine whether $\mathrm{RO}$, atorvastatin and ICI 182,780 exert differential effects on other steroid receptors, we examined their capacity to inhibit transcriptional activity of $\mathrm{AR}$, our rationale being that $\mathrm{AR}$ is also involved in breast cancer progression (10). Among the three ligands tested (RO, atorvastatin and ICI 182,780) only RO inhibited AR-mediated transcription, suggesting that the OSC inhibitor possesses even broader effects than we first anticipated. The ability to block both ER and AR effects possibly indicates that, compared with other chemotherapeutic drugs, RO may have additional advantages with respect to its properties as an anti breast cancer agent.

In our studies using Indigo kits to measure transcriptional activity of nuclear receptors, we utilized non-mammalian cells transfected with human receptor. Following these analyses, we confirmed that RO also affects human breast cancer cells by determining its ability to modify the transcriptional activity of $\mathrm{ER} \alpha$ in human BT-474 cells. This was achieved by assessing the effects of $17 \beta$-estradiol on ER $\alpha$-mediated induction of PR protein, measuring levels of the latter by western blotting. PR is directly regulated by ER $\alpha$ in BT-474 breast cancer cells in a ligand-dependent manner (11). Using western blot analysis, we found that RO suppressed induction of PR by $17 \beta$-estradiol in human BT-474 breast cancer cells (Fig. 6A). Concomitant analysis of ER $\alpha$ mRNA levels showed that under these conditions, RO did not affect expression of ER $\alpha$. This confirms, in human breast cancer cells, that RO has the ability to suppress the biological functions of $\mathrm{ER} \alpha$ without reducing its levels (Fig. 6A and B).

Given that ER $\alpha$-induced signal transduction controls the growth of the majority of breast cancers (16), the results reported in the present study suggest that RO has the potential to be an effective chemotherapeutic agent against hormoneresponsive breast cancer. Based on our observations of its ability to inhibit the biological activities of both ER and AR, further in-depth analysis of the effects of RO on human breast cancer cells is warranted. 


\section{Acknowledgements}

The present study was supported by a Department of Defense Breast Cancer Program grant no. W81XWH-12-1-0191, and by a Faculty Research Grant from the University of Missouri, Columbia. S.M.H. is the Zalk Missouri Professor of Tumor Angiogenesis. Funds to purchase the nanodrop instrument were provided through the generosity of numerous donors to the Ellis Fischel Cancer Center. The authors thank Mr. Jason Lee for his assistance with the figures.

\section{References}

1. Sivente-Poirot S and Poirot M: Cholesterol metabolism and cancer: the good, the bad and the ugly. Curr Opin Pharmacol 12: 673-676, 2012

2. Danilo C and Frank PG: Cholesterol and breast cancer development. Curr Opin Pharmacol 12: 677-682, 2012.

3. Wu Q, Ishikawa T, Sirianni R, Tang H, et al: 27-Hydroxycholesterol promotes cell-autonomous, ER-positive breast cancer. Cell Rep 5: 637-645, 2013.

4. Campagnoli C, Pasanisi P, Castellano I, Abbà C, Brucato T and Berrino F: Postmenopausal breast cancer, androgens, and aromatase inhibitors. Breast Cancer Res Treat 139: 1-11, 2013.

5. Umetani M, Domoto H, Gormley AK, Yuhanna IS, et al: 27-Hydroxycholesterol is an endogenous SERM that inhibits the cardiovascular effects of estrogen. Nat Med 13: 1185-1192, 2007

6. DuSell CD, Umetani M, Shaul PW, Mangelsdorf DJ and McDonnell DP: 27-Hydroxycholesterol is an endogenous selective estrogen receptor modulator. Mol Endocrinol 22: 65-77, 2008
7. Michikawa M and Yanagisawa K: Inhibition of cholesterol production but not of nonsterol isoprenoid products induces neuronal cell death. J Neurochem 72: 2278-2285, 1999.

8. Grinter SZ, Liang Y, Huang SY, Hyder SM and Zou X: An inverse docking approach for identifying new potential anticancer targets. J Mol Graph Model 29: 795-799, 2011.

9. Geisler J, Suzuki T, Helle H, Miki Y, et al: Breast cancer aromatase expression evaluated by the novel antibody 677 correlations to intra-tumor estrogen levels and hormone receptor status. J Steroid Biochem Mol Biol 118: 237-241, 2010.

10. Shah PD, Gucalp A and Traina TA: The role of the androgen receptor in triple-negative breast cancer. Womens Health 9: 351-360, 2013.

11. Saceda M, Grunt TW, Colomer R, Lippman ME, Lupu R and Martin MB: Regulation of estrogen receptor concentration and activity by an erbB/HER ligand in breast carcinoma cell lines. Endocrinology 137: 4322-4330, 1996.

12. Klinge CM: Estrogen receptor interaction with estrogen response elements. Nucleic Acids Res 29: 2905-2919, 2001.

13. Chen L, Monti S, Juszczynski P, Ouyang J, et al: SYK inhibition modulates distinct PI3K/AKT-dependent survival pathways and cholesterol biosynthesis in diffuse large B cell lymphomas. Cancer Cell 23: 826-838, 2013.

14. Fernández C, Martín M, Gómez-Coronado D and Lasunción MA: Effects of distal cholesterol biosynthesis inhibitors on cell proliferation and cell cycle progression. J Lipid Res 46: 920-929, 2005

15. Bachelot T, McCool R, Duffy S, Glanville J, et al: Comparative efficacy of everolimus plus exemestane versus fulvestrant for hormone-receptor-positive advanced breast cancer following progression/recurrence after endocrine therapy: a network meta-analysis. Breast Cancer Res Treat 143: 125-133, 2014.

16. Jensen EV and Jordan VC: The estrogen receptor: a model for molecular medicine. Clin Cancer Res 9: 1980-1989, 2003. 